\title{
ON AN EXTREMAL PROBLEM FOR POLYNOMIALS WITH FIXED MEAN VALUE ${ }^{1}$
}

\author{
Alexander G. Babenko \\ Krasovskii Institute of Mathematics and Mechanics, \\ Ural Branch of the Russian Academy of Sciences; \\ Institute of Mathematics and Computer Science, Ural Federal University, \\ Ekaterinburg, Russia, \\ babenko@imm.uran.ru
}

\begin{abstract}
Let $T_{n}^{+}$be the set of nonnegative trigonometric polynomials $\tau_{n}$ of degree $n$ that are strictly positive at zero. For $0 \leq \alpha \leq 2 \pi /(n+2)$, we find the minimum of the mean value of polynomial $(\cos \alpha-\cos x) \tau_{n}(x) / \tau_{n}(0)$ over $\tau_{n} \in T_{n}^{+}$on the period $[-\pi, \pi)$.
\end{abstract}

Key words: Trigonometric polynomials, Extremal problem.

Let $T_{n}$ be the space of trigonometric polynomials of degree $n$ with real coefficients, and let $T_{n}^{+}$ be the set of nonnegative polynomials from $T_{n}$ that are strictly positive at zero. For a real $\alpha$ we define

$$
\chi_{n}(\alpha)=\inf _{\tau_{n} \in T_{n}^{+}} \frac{1}{2 \pi \tau_{n}(0)} \int_{-\pi}^{\pi} \tau_{n}(x)(\cos \alpha-\cos x) d x .
$$

In 1915, Fejér [4] (see also [2, vol. 2, Sec. 6, Problem 52]) proved the following statement.

Fejér's Theorem. Let the polynomial $\tau_{n}(x)=a_{0}+\sum_{\nu=1}^{n}\left(a_{\nu} \cos \nu x+b_{\nu} \sin \nu x\right)$ belong to the set $T_{n}^{+}$. Then

$$
\sqrt{a_{1}^{2}+b_{1}^{2}} \leq 2 a_{0} \cos \frac{\pi}{n+2} .
$$

This inequality turns into the equality for the polynomial

$$
t_{n}(x)=\left(\cos \frac{n+2}{2} x\right)^{2} /\left(\cos x-\cos \frac{\pi}{n+2}\right)^{2} .
$$

This theorem is equivalent to the statement that

$$
\chi_{n}(\pi /(n+2))=0 .
$$

For $0 \leq \alpha<\pi$, put

$$
\begin{gathered}
Q_{(n+3) / 2, \alpha}(x)=\left(\sin \frac{n+1}{2} \alpha \sin \frac{n+3}{2} x-\sin \frac{n+3}{2} \alpha \sin \frac{n+1}{2} x\right) / \sin \frac{\alpha}{2}, \quad 0<\alpha<\pi, \\
Q_{(n+3) / 2,0}(x)=\lim _{\alpha \rightarrow 0} Q_{(n+3) / 2, \alpha}(x)=(n+1) \sin \frac{n+3}{2} x-(n+3) \sin \frac{n+1}{2} x .
\end{gathered}
$$

In this paper we prove the following result.

\footnotetext{
${ }^{1}$ The paper was originally published in a hard accessible collection of articles Approximation of Functions by Polynomials and Splines (The Ural Scientific Center of the Academy of Sciences of the USSR, Sverdlovsk, 1985), p. 15-22 (in Russian).
} 
Theorem. Let $n$ be a nonnegative integer and $0 \leq \alpha \leq 2 \pi /(n+2)$. Then (1) takes the value

$$
\begin{gathered}
\chi_{n}(\alpha)=\frac{\left(\sin \frac{n+3}{2} \alpha-\sin \frac{n+1}{2} \alpha\right)(1-\cos \alpha)}{(n+3) \sin \frac{n+1}{2} \alpha-(n+1) \sin \frac{n+3}{2} \alpha}, \quad 0<\alpha \leq \frac{2 \pi}{n+2}, \\
\chi_{n}(0)=\lim _{\alpha \rightarrow 0} \chi_{n}(\alpha)=\frac{6}{(n+1)(n+2)(n+3)},
\end{gathered}
$$

and the infimum is attained for the polynomial

$$
\tau_{n, \alpha}(x)=\left(\frac{Q_{(n+3) / 2, \alpha}(x)}{(\cos x-\cos \alpha) \sin (x / 2)}\right)^{2},
$$

where $Q_{(n+3) / 2, \alpha}$ is given by (5).

Note that $\chi_{n}(\alpha) \geq 0$ for $0 \leq \alpha \leq \pi /(n+2)$ and $\chi_{n}(\alpha) \leq 0$ for $\pi /(n+2) \leq \alpha \leq 2 \pi /(n+2)$. First we prove two auxiliary statements. Set $\alpha_{0}=\pi, \alpha_{1}=2 \pi / 3$, and for $n \geq 2$ let $\alpha_{n}$ be the first positive root of the equation

$$
\left(\sin \frac{n+3}{2} x\right) / \sin \frac{n+1}{2} x=c_{n}, \quad c_{2 m}=-1, \quad c_{2 m-1}=-\frac{m+1}{m} .
$$

It is easy to see that for $r \geq 2$ we have

$$
\alpha_{2 r-2}=\pi / r, \quad 2 \pi /(2 r+1)<\alpha_{2 r-1}<\pi / r .
$$

Lemma 1. If $n$ is a nonnegative integer and $0<\alpha<\alpha_{n}$, then the function $Q_{(n+3) / 2, \alpha}$ defined by (5) has exactly $[(n+5) / 2]$ zeros $x_{0}=0<x_{1}=\alpha<x_{2}<x_{3}<\ldots<x_{[(n+3) / 2]}$ in the interval $[0, \pi]$. For each polynomial $\tau_{n+1} \in T_{n+1}$ we have

$$
\begin{gathered}
\frac{1}{2 \pi} \int_{-\pi}^{\pi} \tau_{n+1}(x) d x=\frac{\sin \frac{n+1}{2} \alpha-\sin \frac{n+3}{2} \alpha}{(n+3) \sin \frac{n+1}{2} \alpha-(n+1) \sin \frac{n+3}{2} \alpha} \tau_{n}(0) \\
+\sum_{k=1}^{[(n+3) / 2]} g_{n+1}\left(x_{k}\right)\left(\tau_{n+1}\left(x_{k}\right)+\tau_{n+1}\left(-x_{k}\right)\right),
\end{gathered}
$$

where

$$
\begin{gathered}
g_{2 r-1}(x)=\frac{\sin x}{2 r \sin x-\sin 2 r x}, \\
g_{2 r}(x)= \begin{cases}\frac{\sin x}{2(r \sin x-\sin r x \cos (r+1) x)}, & x \neq \pi, \\
\frac{\sin r \alpha+\sin (r+1) \alpha}{4(r \sin (r+1) \alpha+(r+1) \sin r \alpha)}, & x=\pi .\end{cases}
\end{gathered}
$$

Moreover, the numbers $\left(\frac{2 \pi}{n+2}-\alpha\right) g_{n+1}\left(x_{[(n+3) / 2]}\right), g_{n+1}\left(x_{k}\right), 1 \leq k \leq[(n+1) / 2]$, are nonnegative.

P r o o f. First we consider the case when $n=0$ and $0<\alpha<\pi$. The function $Q_{3 / 2, \alpha}(x)=$ $2 \sin (x / 2)(\cos x-\cos \alpha)$ has two zeros $x_{0}=0, x_{1}=\alpha$ in the interval $[0, \pi]$. We have

$$
\frac{1}{2 \pi} \int_{-\pi}^{\pi} \tau_{1}(x) d x=\frac{\cos \alpha}{\cos \alpha-1} \tau_{1}(0)+\frac{\tau_{1}(\alpha)+\tau_{1}(-\alpha)}{2(1-\cos \alpha)}, \quad \tau_{1} \in T_{1},
$$

since this formula is valid for the polynomials $1, \sin x, \cos x$ and thus the lemma follows for $n=0$.

Now let $n=1$ and $0<\alpha<2 \pi / 3$. Then the function $Q_{2, \alpha}(x)=4 \cos \frac{\alpha}{2} \sin x(\cos x-\cos \alpha)$ has three zeros $x_{0}=0, x_{1}=\alpha, x_{2}=\pi$ in the interval $[0, \pi]$. The quadrature formula

$$
\frac{1}{2 \pi} \int_{-\pi}^{\pi} \tau_{2}(x) d x=\frac{1-2 \cos \alpha}{4(1-\cos \alpha)} \tau_{2}(0)+\frac{\tau_{2}(\alpha)+\tau_{2}(-\alpha)}{2(1-\cos \alpha)}+\frac{1+2 \cos \alpha}{8(1+\cos \alpha)}\left(\tau_{2}(\pi)+\tau_{2}(-\pi)\right), \quad \tau_{2} \in T_{2},
$$


holds, for it holds for the polynomials $\sin x, \sin 2 x,(1+\cos x)(\cos \alpha-\cos x), 1-\cos 2 x$, $(1-\cos x)(\cos \alpha-\cos x)$ which generate the space $T_{2}$. This proves the lemma for $n=1$.

Next we consider the case of an odd $n=2 r-1, r \geq 2$, and $0<\alpha<\alpha_{2 r-1}$. The function (5) can be written in the form $Q_{r+1, \alpha}(x)=(\cos x-\cos \alpha) \sin x S_{r-1, \alpha}(x) / \sin (\alpha / 2)$, where

$$
S_{r-1, \alpha}(x)=\frac{\sin r \alpha \sin (r+1) x-\sin (r+1) \alpha \sin r x}{(\cos x-\cos \alpha) \sin x}
$$

is a cosine polynomial of degree $r-1$. To study the zeros of the polynomial $S_{r-1, \alpha}$, we write it in the form $S_{r-1, \alpha}(x)=\frac{(f(x)-f(\alpha)) \sin r \alpha \sin r x}{\sin x(\cos x-\cos \alpha)}$, where $f(x)=\frac{\sin (r+1) x}{\sin r x}$. When $x$ runs over the intervals $(0, \pi / r),((r-1) \pi / r, \pi)$, and $(k \pi / r,(k+1) \pi / r), 1 \leq k \leq r-2$, then the values of $f$ run continuously over the intervals $((r+1) / r,-\infty),(+\infty,-(r+1) / r)$, and $(+\infty,-\infty)$, respectively. Thus, taking into account the definition (8) of $\alpha_{2 r-1}$, we see that for each $\alpha$ in the interval $\left(0, \alpha_{2 r-1}\right)$ the polynomial $S_{r-1, \alpha}$ has exactly $r-1$ zeros $x_{2}<x_{3}<\ldots<x_{r}$ in the interval $(\alpha, \pi)$. Moreover, these zeros are all simple since $S_{r-1, \alpha}$ has degree $r-1$. It is known [3, p. 403, formulae 30, 31, 33] that

$$
\frac{1}{\pi} \int_{0}^{\pi} \frac{\sin m x \cos \nu x}{\sin x} d x=\left\{\begin{array}{l}
1, m>\nu, m+\nu=2 k-1 \\
0, m>\nu, m+\nu=2 k \\
0, m \leq \nu
\end{array}\right.
$$

It follows that for the polynomial (12) we have

$$
\frac{1}{\pi} \int_{0}^{\pi} S_{r-1, \alpha}(x) \cos \nu x(1+\cos x)(\cos \alpha-\cos x) d x=\sin (r+1) \alpha-\sin r \alpha, \quad \nu=0,1, \ldots, r-1 .
$$

Consequently, for each cosine polynomial $C_{r-1}$ of degree $r-1$ we have

$$
\frac{1}{\pi} \int_{0}^{\pi} S_{r-1, \alpha}(x) C_{r-1}(x)(1+\cos x)(\cos \alpha-\cos x) d x=(\sin (r+1) \alpha-\sin r \alpha) C_{r-1}(0) .
$$

Thus, the polynomial $S_{r-1, \alpha}$ is orthogonal with the weight $(\cos x-\cos \alpha)(1-\cos x)(1+\cos x)$ to all cosine polynomials of degree $r-2$.

We will need the following known result (e.g., [1, pp. 162, 163]). Let the weight $v(x)$ and the points $a_{1}, \ldots, a_{m}$ in the interval $[0, \pi]$ be given. A quadrature formula of the form

$$
\int_{0}^{\pi} C_{2 \nu+m-1}(x) v(x) d x=\sum_{\ell=1}^{m} A_{\ell} C_{2 \nu+m-1}\left(a_{\ell}\right)+\sum_{k=1}^{\nu} B_{k} C_{2 \nu+m-1}\left(x_{k}\right)
$$

which is exact for cosine polynomials of degree $2 \nu+m-1$ exists if and only if there exists a cosine polynomial $S_{\nu}$ of degree $\nu$ which is orthogonal to all cosine polynomials of degree $\nu-1$ with the weight $v(x)\left(\cos x-\cos a_{1}\right) \ldots\left(\cos x-\cos a_{m}\right)$. The zeros of the polynomial $S_{\nu}$ coincide with the nodes $x_{1}, x_{2}, \ldots, x_{\nu}$; they should be all distinct and differ from the fixed nodes $a_{1}, \ldots, a_{m}$.

By this result, there exist numbers $\varepsilon_{0}, \ldots, \varepsilon_{r+1}$ such that for each cosine polynomial $C_{2 r}$ of degree $2 r$ we have

$$
\frac{1}{\pi} \int_{0}^{\pi} C_{2 r}(x) d x=\sum_{k=0}^{r+1} \varepsilon_{k} C_{2 r}\left(x_{k}\right)
$$

where $x_{2}, x_{3}, \ldots, x_{r}$ are the zeros of the polynomial $S_{r-1, \alpha}$ in the interval $(\alpha, \pi), x_{0}=0, x_{1}=\alpha$, $x_{r+1}=\pi$.

Note that, for $\nu=1,2, \ldots, r$, the zeros of the polynomial

$$
S_{r-1, x_{\nu}}(x)=\frac{\sin r x_{\nu} \sin (r+1) x-\sin (r+1) x_{\nu} \sin r x}{\left(\cos x-\cos x_{\nu}\right) \sin x}
$$


coincide with the zeros of the polynomial $(\cos x-\cos \alpha) S_{r-1, \alpha}(x) /\left(\cos x-\cos x_{\nu}\right)$. Thus,

$$
S_{r-1, x_{\nu}}(x)=\mathcal{A}_{\nu}(\cos x-\cos \alpha) S_{r-1, \alpha}(x) /\left(\cos x-\cos x_{\nu}\right),
$$

where $\mathcal{A}_{\nu}$ is a constant that does not depend on $x$.

It is not difficult to check that, for $\nu=1,2, \ldots, r$, the polynomial (16) satisfies the equations

$$
S_{r-1, x_{\nu}}(x)=\frac{D_{r}\left(x-x_{\nu}\right)-D_{r}\left(x+x_{\nu}\right)}{2 \sin x}=2 \sum_{k=1}^{r} \frac{\sin k x_{\nu} \sin k x}{\sin x},
$$

where

$$
D_{r}(x)=1+2 \sum_{k=1}^{r} \cos k x=\frac{\sin \frac{2 r+1}{2} x}{\sin (x / 2)}
$$

is the Dirichlet kernel.

Using (18), we obtain

$$
\frac{1}{\pi} \int_{0}^{\pi} S_{r-1, x_{\nu}}(x)(\sin x)^{2} d x=\sin x_{\nu}, \quad \nu=1,2, \ldots, r .
$$

Using (19), (17) and (18), one can calculate the following coefficients of the quadrature formula (15):

$$
\varepsilon_{\nu}=1 /\left(2 \sum_{k=1}^{r}\left(\sin k x_{\nu}\right)^{2}\right)=\frac{\sin x_{\nu}}{r \sin x_{\nu}-\sin r x_{\nu} \cos (r+1) x_{\nu}}, \quad 1 \leq \nu \leq r .
$$

By (14), we have

$$
\frac{1}{\pi} \int_{0}^{\pi} S_{r-1, \alpha}(x)(1+\cos x)(\cos \alpha-\cos x) d x=\sin (r+1) \alpha-\sin r \alpha .
$$

Using (15) and (12), we obtain from here that

$$
\varepsilon_{0}=\frac{\sin r \alpha-\sin (r+1) \alpha}{2((r+1) \sin r \alpha-r \sin (r+1) \alpha)} .
$$

By (13) and (12) we conclude that

$$
\frac{1}{\pi} \int_{0}^{\pi} S_{r-1, \alpha}(x)(1-\cos x)(\cos x-\cos \alpha) d x=(-1)^{r}(\sin r \alpha+\sin (r+1) \alpha) .
$$

Formulae (22), (15) and (12) imply

$$
\varepsilon_{r+1}=\frac{\sin r \alpha+\sin (r+1) \alpha}{2((r+1) \sin r \alpha+r \sin (r+1) \alpha)} .
$$

It is easy to check that

$$
\left(\frac{2 \pi}{2 r+1}-\alpha\right) \varepsilon_{r} \geq 0
$$

for $0<\alpha<\alpha_{2 r-1}$. The statement of the lemma for $n=2 r-1, r \geq 2$, now follows from (20), (21), (23) and (24).

Finally, let us consider the case when $n=2 r-2, r \geq 2$, and $0<\alpha<\pi / r$. Function (5) can be written in the form $Q_{(2 r+1) / 2, \alpha}(x)=\sin (x / 2)(\cos x-\cos \alpha) \Theta_{r-1, \alpha}(x) / \sin (\alpha / 2)$, where

$$
\Theta_{r-1, \alpha}(x)=\frac{\sin \frac{2 r-1}{2} \alpha \sin \frac{2 r+1}{2} x-\sin \frac{2 r+1}{2} \alpha \sin \frac{2 r-1}{2} x}{(\cos x-\cos \alpha) \sin (x / 2)}=\frac{(\varphi(x)-\varphi(\alpha)) \sin \frac{2 r-1}{2} \alpha \sin \frac{2 r-1}{2} x}{(\cos x-\cos \alpha) \sin (x / 2)}
$$


here, $\varphi(x)=\left(\sin \frac{2 r+1}{2} x\right) / \sin \frac{2 r-1}{2} x$. When $x$ runs over the intervals $(0,2 \pi /(2 r-1)),(2(r-1) \pi /(2 r-$ $1), \pi)$ and $(2 k \pi /(2 r-1), 2(k+1) \pi /(2 r-1)), 1 \leq k \leq r-2$, then the values of the function $\varphi$ run continuously over the intervals $((2 r+1) /(2 r-1),-\infty),(+\infty,-1)$ and $(+\infty,-\infty)$, respectively. Thus, for $0<\alpha<\pi / r$ the polynomial $\Theta_{r-1, \alpha}$ has exactly $r-1$ simple zeros $x_{2}<x_{3}<\ldots<x_{r}$ in the interval $(\alpha, \pi)$. With the help of (13) and (25), repeating the arguments used in the proof of formula (14), we see that

$$
\frac{1}{\pi} \int_{0}^{\pi} \Theta_{r-1, \alpha}(x) C_{r-1}(x)(\cos \alpha-\cos x) d x=\left(\sin \frac{2 r+1}{2} \alpha-\sin \frac{2 r-1}{2} \alpha\right) C_{r-1}(0)
$$

for all cosine polynomials $C_{r-1}$ of degree $r-1$. Thus, the polynomial $\Theta_{r-1, \alpha}$ is orthogonal to all cosine polynomials of degree $r-2$ with the weight $(1-\cos x)(\cos x-\cos \alpha)$. It follows that there exist numbers $\delta_{0}, \delta_{1}, \ldots, \delta_{r}$ such that the quadrature formula

$$
\frac{1}{\pi} \int_{0}^{\pi} C_{2 r-1}(x) d x=\sum_{k=0}^{r} \delta_{k} C_{2 r-1}\left(x_{k}\right),
$$

where $x_{2}, x_{3}, \ldots, x_{r}$ are the zeros of the polynomial $\Theta_{r-1, \alpha}$ in the interval $(\alpha, \pi), x_{0}=0, x_{1}=\alpha$, is exact for all cosine polynomials $C_{2 r-1}$ of degree $2 r-1$.

Note that, for $\nu=1,2, \ldots, r$, the polynomial

$$
\Theta_{r-1, x_{\nu}}(x)=\frac{\sin \frac{2 r-1}{2} x_{\nu} \sin \frac{2 r+1}{2} x-\sin \frac{2 r+1}{2} x_{\nu} \sin \frac{2 r-1}{2} x}{\left(\cos x-\cos x_{\nu}\right) \sin (x / 2)}
$$

satisfies the equation

$$
\Theta_{r-1, x_{\nu}}(x)=\mathcal{B}_{\nu}(\cos x-\cos \alpha) \Theta_{r-1, \alpha}(x) /\left(\cos x-\cos x_{\nu}\right), \quad 1 \leq \nu \leq r,
$$

where $\mathcal{B}_{\nu}$ is a constant that does not depend on $x$.

Moreover, the polynomial (28) can be rewritten in the form

$$
\Theta_{r-1, x_{\nu}}(x)=2 \sum_{k=1}^{r}\left(\sin \frac{2 k-1}{2} x_{\nu} \sin \frac{2 k-1}{2} x\right) / \sin \frac{x}{2} .
$$

This implies the equation

$$
\frac{1}{\pi} \int_{0}^{\pi} \Theta_{r-1, x_{\nu}}(x)\left(\sin \frac{x}{2}\right)^{2} d x=\sin \frac{x_{\nu}}{2}, \quad 1 \leq \nu \leq r .
$$

Formulae (31), (27), (29) and (30) yield

$$
\delta_{\nu}=1 /\left(2 \sum_{k=1}^{r}\left(\sin \frac{2 k-1}{2} x_{\nu}\right)^{2}\right)=\frac{2 \sin x_{\nu}}{2 r \sin x_{\nu}-\sin 2 r x_{\nu}}, \quad 1 \leq \nu \leq r .
$$

By (26) we obtain

$$
\frac{1}{\pi} \int_{0}^{\pi} \Theta_{r-1, x_{\nu}}(x)(\cos \alpha-\cos x) d x=\sin \frac{2 r+1}{2} \alpha-\sin \frac{2 r-1}{2} \alpha .
$$

Using (33), (27) and (25), we get

$$
\delta_{0}=\left(\sin \frac{2 r-1}{2} \alpha-\sin \frac{2 r+1}{2} \alpha\right) /\left((2 r+1) \sin \frac{2 r-1}{2} \alpha-(2 r-1) \sin \frac{2 r+1}{2} \alpha\right) .
$$

The statement of the lemma for $n=2 r-2, r \geq 2$, now follows from (32) and (34). This completes the proof of the lemma. 
Lemma 2. Let $n$ be a nonnegative integer, $0 \leq \alpha \leq \alpha_{n}$ if $n$ is even and $0 \leq \alpha<\alpha_{n}$ if $n$ is odd. For each polynomial $\tau_{n} \in T_{n}$ we have

$$
\begin{gathered}
\frac{1}{2 \pi} \int_{-\pi}^{\pi} \tau_{n}(x)(\cos \alpha-\cos x) d x=\frac{\left(\sin \frac{n+3}{2} \alpha-\sin \frac{n+1}{2} \alpha\right)(1-\cos \alpha)}{(n+3) \sin \frac{n+1}{2} \alpha-(n+1) \sin \frac{n+3}{2} \alpha} \tau_{n}(0) \\
+\sum_{k=1}^{[(n+1) / 2]} g_{n+1}\left(x_{k}\right)\left(\cos \alpha-\cos x_{k}\right)\left(\tau_{n}\left(x_{k}\right)+\tau_{n}\left(-x_{k}\right)\right)
\end{gathered}
$$

where $x_{1}<x_{2}<\cdots<x_{[(n+1) / 2]}$ are the zeros of the polynomial $(7)$ in the interval $(\alpha, \pi]$, and the numbers $g_{n+1}\left(x_{k}\right), k=1,2, \ldots,[(n+1) / 2]$, are defined by equations (11). Moreover, the coefficients $g_{n+1}\left(x_{k}\right)\left(\cos \alpha-\cos x_{k}\right), k=1,2, \ldots,[(n-1) / 2]$, are nonnegative, as well as the number $\left(\frac{2 \pi}{n+2}-\alpha\right) g_{n+1}\left(x_{[(n+1) / 2]}\right)\left(\cos \alpha-\cos x_{[(n+1) / 2]}\right)$.

$\mathrm{Pr}$ o o f. For $0<\alpha<\alpha_{n}$, the statement is a straightforward consequence of Lemma 1 . Let $\tau_{n}$ be an arbitrary polynomial of degree $n$, then the right-hand side of (35) and the coefficients of this quadrature formula tend uniformly to the claimed (bounded) values as $\alpha \rightarrow 0$, and the statement of the lemma follows for $\alpha=0$. The case of $\alpha=\alpha_{n}$ with even $n$ can be proved in a similar way. As for the case of odd $n$, note that for an odd $n \geq 3$ we have $g_{n+1}\left(x_{[(n+1) / 2]}\right)\left(\cos \alpha-\cos x_{[(n+1) / 2]}\right)=$ $g(\pi)(\cos \alpha+1) \rightarrow-\infty$ as $\alpha \rightarrow \alpha_{n}$, while $g_{n+1}\left(x_{[(n-1) / 2]}\right)\left(\cos \alpha-\cos x_{[(n-1) / 2]}\right) \rightarrow+\infty$ as $\alpha \rightarrow \alpha_{n}$.

$\mathrm{P}$ r o of of the theorem. The statement of the theorem follows from the fact that for each nonnegative polynomial $\tau_{n}$ and each number $\alpha$ in the interval $[0,2 \pi /(n+2)]$ we have, by Lemma 2 , the inequality

$$
\frac{1}{2 \pi} \int_{-\pi}^{\pi} \tau_{n}(x)(\cos \alpha-\cos x) d x \geq \frac{\left(\sin \frac{n+3}{2} \alpha-\sin \frac{n+1}{2} \alpha\right)(1-\cos \alpha)}{(n+3) \sin \frac{n+1}{2} \alpha-(n+1) \sin \frac{n+3}{2} \alpha} \tau_{n}(0) .
$$

This inequality turns into the equality for the polynomial $\tau_{n, \alpha}$. This proves the theorem.

\section{Acknowledgments}

The author is grateful to Professor V. V. Arestov for the statement of the problem as well as to Doctor E. E. Berdysheva for the excellent translation of the paper into English.

\section{REFERENCES}

1. Krylov V. I. Approximate calculation of integrals. Fizmatgiz, Moscow. 1959. [Russian]

2. Pólya, G., Szegö, G. Problems and theorems in analysis. Springer, Berlin. Vol. 2. 1998.

3. Prudnikov, A.P., Brychkov, Yu. A., Marichev, O. I. Integrals and series. Nauka, Moscow. 1981. [Russian]

4. Fejér L. Über trigonometrische Polynome. Gesammelte Arbeit, Bd. 1, Akad. Kiado, Budapest. 1970. P. 842-872. 